\title{
Role of posaconazole in the treatment of oropharyngeal candidiasis
}

REVIEW

This article was published in the following Dove Press journal:

Infection and Drug Resistance

26 June 2010

Number of times this article has been viewed

\author{
Voichita lanas' \\ Kathryn R Matthias ${ }^{2}$ \\ Stephen A Klotz' \\ 'Section of Infectious Diseases and \\ Department of Medicine, ${ }^{2}$ School \\ of Pharmacy, University of Arizona, \\ Tucson, Arizona, USA
}

\begin{abstract}
Posaconazole is the newest azole antifungal approved by the US Food and Drug Administration, and possesses a broad spectrum of activity against numerous yeasts and filamentous fungi. It is available as an oral suspension and is generally well tolerated by patients, but gastrointestinal absorption is sometimes inadequate and remains a clinical concern in treating deep-seated infections. It is used routinely and effectively for the prophylaxis of invasive fungal infections in immunosuppressed hosts and is an effective treatment of oropharyngeal candidiasis, including azole-resistant disease.
\end{abstract}

Keywords: posaconazole, azole, yeasts, filamentous fungi

\section{Overview of oropharyngeal candidiasis}

The frequency of Candida species infections in humans continues to increase. There are many manifestations of the disease, some trivial and some life-threatening. The most common candidal infection is mucocutaneous oropharyngeal candidiasis (OPC). Thrush is the classic lesion of OPC and is recognized by the presence of creamy white, curd-like patches on the soft and hard palate and other oral mucosal surfaces. Diagnosis is confirmed by scraping one of the lesions and using potassium hydroxide or Gram stain of scraped material which on microscopy demonstrates hyphae, pseudohyphae, and/or yeast forms with scattered polymorphonuclear leukocytes. The fungi are usually entangled with Gram positive cocci. ${ }^{1}$ Other manifestations of OPC include atrophy of the tongue or acute atrophic candidiasis, "denture sore mouth" or chronic atrophic candidiasis (inflammation under the dental plates), angular cheilitis (inflammation at the corners of the mouth), and Candida leukoplakia (firm, white patches, persistently present on the oral mucosa). ${ }^{2}$

The Candida species commonly associated with OPC is C. albicans. ${ }^{2}$ In human immunodeficiency virus (HIV)-positive individuals, C. albicans is isolated in over $70 \%$ of the patients, and less often C. glabrata, C. tropicalis, C. parapsilosis, ${ }^{3}$ or $C$. dublinensis are found. ${ }^{4} \mathrm{OPC}$ is common in HIV-positive patients with CD4 counts lower than 200 cells $/ \mu \mathrm{L}$, immunocompromised patients on chemotherapy, patients on systemic steroids, or patients who use inhaled steroids. Approximately $80 \%-90 \%$ of patients with acquired immunodeficiency syndrome (AIDS) will develop OPC. ${ }^{5}$

Preferred therapy for OPC differs by patient population. For example, therapy in the HIV patient may differ from that in a patient with immunosuppression secondary to steroids or chemotherapy. In HIV-negative patients with mild OPC, treatment consists of
Correspondence: Stephen A Klotz Section of Infectious Diseases, I50I N Campbell Ave, Tucson, Arizona 85724, USA

Tel +I 5206266887

Fax + I 5206265183

Email sklotz@u.arizona.edu 
local antifungal lozenges or solutions, eg, nystatin swish and swallow four times a day or clotrimazole troches (one $10 \mathrm{mg}$ troche five times daily). If local therapy does not work, oral therapy is recommended, such as fluconazole $200 \mathrm{mg}$ once daily, then 100-200 mg daily. ${ }^{6}$ These recommendations are derived from a consensus of authorities, with moderate evidence for or against the use of a regimen based upon more than one well-designed clinical study. ${ }^{6}$ Recurrence is common if underlying risk factors are still present (eg, continued use of steroids or chemotherapy). In HIVpositive patients with OPC, treatment also varies depending on the severity of disease. Topical agents can be used in mild OPC, but oral azoles are indicated in moderate to severe OPC. Antiretroviral drugs are essential in the treatment and eradication of OPC in AIDS patients; if antiretrovirals are not started, the rate of relapse is high. Topical treatment has no role if esophageal candidiasis is present. ${ }^{6}$

\section{Microbiological aspects of posaconazole}

Posaconazole is a triazole antifungal agent that has structural similarities to itraconazole. Posaconazole inhibits the synthesis of ergosterol (a sterol component of fungal cell membranes) by inhibition of the enzyme, lanosterol 14-alpha demethylase, with accumulation of methylated sterol precursors. ${ }^{7}$ In contrast with other azole antifungals, posaconazole is not extensively metabolized by the CYP450 enzymes. ${ }^{8}$ In vitro tests have shown posaconazole to be active against Candida species, Aspergillus species, Coccidioides species, Fusarium species, Histoplasma capsulatum, Zygomycetes, Phaeohyphomycetes, and other filamentous fungi. In addition, posaconazole has demonstrated activity against fluconazole- and itraconazole-resistant Candida species, as well as itraconazole-, voriconazole-, and amphotericin B-resistant Aspergillus fumigatus, and activity against Cryptococcus neoformans. ${ }^{9}$ Posaconazole is fungistatic against some Candida species (C. albicans, C. glabrata, C. parapsilosis, and C. tropicalis), but is fungicidal against other Candida species (C. Iusitaniae, C. krusei, C. kefyr, and C. inconspicua). ${ }^{10}$ Clinical uses of posaconazole that are Food and Drug Administration (FDA)-approved include prophylaxis of invasive aspergillosis in high-risk patients, prophylaxis of disseminated candidiasis in severely immunocompromised patients, and treatment of OPC, including the treatment of OPC refractory to itraconazole and/or fluconazole. Clinical applications that are not FDA-approved include invasive aspergillosis or esophageal candidiasis.

\section{OPC treatment and a comparator drug, fluconazole}

Fluconazole is a triazole with greater than $90 \%$ bioavailability after oral administration and has been extensively used for treatment of a wide range of Candida infections, in particular, OPC. ${ }^{6}$ Although OPC usually responds to fluconazole, if underlying predisposing factors are not corrected, OPC can recur. There have been a number of reports of fluconazole failure or resistance in AIDS patients. ${ }^{11}$ However, it is difficult to establish a definition of resistance. In some cases, the term is used when a patient fails to respond clinically to antifungal therapy, and in other cases, resistance is described by the minimum inhibitory concentration (MIC) of the drug. While correlations between the fluconazole MIC and outcome have been established in animal models, therapeutic failures and successes in humans may be seen with isolates for which MICs are both high and low. ${ }^{12}$ MIC breakpoints for fluconazole remain unclear, but the Clinical and Laboratory Standards Institute methodology recommends the following breakpoints: susceptible (S) MIC $\leq 8 \mu \mathrm{L}$; susceptible, dose-dependent (SDD) MIC 16-32 $\mu \mathrm{L}$ that predicts a poor response to $100 \mathrm{mg}$ a day of fluconazole orally; or resistant (R) $\mathrm{MIC}>64 \mu \mathrm{L} .{ }^{13}$ On the basis of previously published data, it appears that resistance occurs in roughly $5 \%$ of patients with advanced AIDS. ${ }^{3}$ The largest group of failures reported outside of patients with AIDS and OPC consists of patients with fungemia. In both groups, one of the major factors predicting fluconazole resistance is low-dose fluconazole used as prophylaxis. ${ }^{12}$

The clinical significance of fluconazole resistance as defined in the laboratory is not fully known. Most OPC cases described in the literature of fluconazole resistance have been in AIDS patients. The clinical significance of fluconazole resistance was assessed in $50 \mathrm{HIV}$-positive patients with recurrent OPC in a study by Revankar et al. ${ }^{14}$ Resistant yeast was defined as an MIC $>8 \mu \mathrm{L}$. Thirty-two of 50 patients were found to have resistant Candida (11 had MIC $>32 \mu \mathrm{L}$ ), but almost all patients responded clinically to fluconazole (48/50), which suggests that the clinical efficacy of fluconazole remains high. ${ }^{14}$

\section{Resistance issues: azole-resistant Candida species}

Several mechanisms give rise to resistance to azole drugs. One is mutation in the gene, ERG11, which encodes the target enzyme of azoles, ie, lanosterol demethylase. Another resistant mechanism of Candida species is overexpression of drug 
efflux pumps. Posaconazole, however, is not a substrate for two major pumps, MDR1 and FLU1, that confer resistance to voriconazole or fluconazole. ${ }^{15}$ In fact, posaconazole may have clinical activity against fluconazole-resistant Candida species. ${ }^{11}$ Posaconazole has good activity against Candida species that are less susceptible to fluconazole (eg, C. krusei, C. guilliermondii, and C. dublinensis), but is less active against $C$. glabrata. ${ }^{9}$ Based on $\mathrm{MIC}_{90}$ values, posaconazole was found to be more potent than fluconazole and equal or more potent than itraconazole, voriconazole, and amphotericin B against these isolates. ${ }^{9}$ However, more than half of Candida isolates resistant to fluconazole were also less susceptible to posaconazole. ${ }^{16}$ The clinical significance of these findings is unknown. ${ }^{10}$

\section{Pharmacology and pharmacokinetics of posaconazole}

Guidelines developed by the Infectious Diseases Society of America for candidiasis recommend posaconazole for fluconazole-refractory OPC at a dose of $400 \mathrm{mg}$ orally twice daily for three days, followed by $400 \mathrm{mg}$ orally once daily for up to 28 days. $^{6}$ However, the manufacturer's dosing information is slightly different. For treatment of OPC, the loading dose is $100 \mathrm{mg}(2.5 \mathrm{~mL})$ orally twice daily for one day, then $100 \mathrm{mg}$ orally once daily for 13 days. The manufacturer's recommendation for treatment of OPC refractory to itraconazole and fluconazole is $400 \mathrm{mg}(10 \mathrm{~mL})$ twice a day for a duration based on the severity of the disease and clinical response. ${ }^{17}$

Posaconazole is given orally. The oral solution is administered during or within 20 minutes of a full meal or a liquid nutritional supplement. Alternatively, the drug can be administered with an acidic carbonated drink. It should be stored at $25^{\circ} \mathrm{C}$. There is no dose adjustment recommended for renal or liver failure. ${ }^{8,18}$ The structural formula is shown in Figure 1.

Following multiple dose administration, steady-state plasma concentrations are attained at 7-10 days. During twice daily dosing of posaconazole over 14 days, mean peak plasma concentrations were achieved on day 1 at five hours and eight

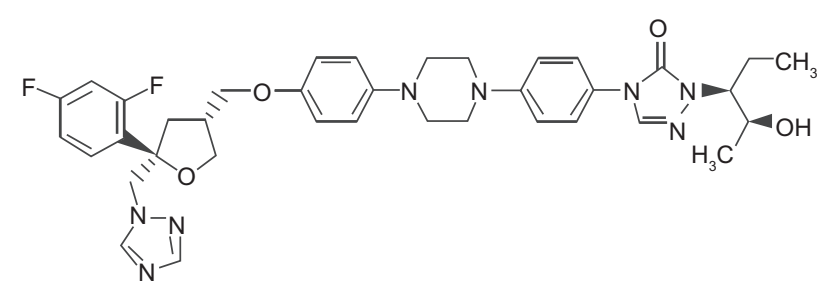

Figure I Structural formula of posaconazole. hours following the administration of the first and second dose, respectively. On day 14 , peak plasma concentrations were observed at 4-6 hours and at 9-11 hours following the administration of the first and second doses, respectively. ${ }^{19}$ Absorption is influenced by the frequency of doses and meals. Posaconazole is absorbed better if taken with meals, especially high-fat meals. Acidic carbonated beverages also increase absorption. ${ }^{20}$

Posaconazole is $98 \%$ protein-bound, mostly to albumin. It is lipophilic and has a high volume of distribution suggesting extensive extravascular distribution and penetration into body fluids, bone, central nervous system, eye, and pulmonary epithelial lining fluid. A very small percentage of posaconazole is metabolized and the majority of the drug is eliminated from the body unchanged. Less than $30 \%$ is metabolized by the liver through glucuronidation and is transformed into biologically inactive metabolites. ${ }^{21}$ The majority of posaconazole is excreted in the feces up to 120 hours after a dose. Only $13 \%-14 \%$ of posaconazole is excreted in the urine. ${ }^{18}$ Mean total body clearance is $32 \mathrm{~L} /$ hour. The elimination half-life of the parent compound is 35 hours, with a range of 20-66 hours. ${ }^{20}$

\section{Efficacy studies in OPC}

The clinical efficacy of posaconazole in OPC was investigated in two major studies done in HIV-positive patients. In a multicenter, randomized, evaluator-blinded trial by Vasquez et al in 2006, posaconazole was compared with fluconazole in the treatment of OPC in HIV-infected patients. ${ }^{22}$ The primary endpoint was clinical success (cure or improvement) on day 14. This was evaluated in 329 patients. Other secondary endpoints were mycologic success, defined as a quantitative yeast culture yielding 20 colony forming units (cfu)/mL of Candida species and eradication, defined as $0 \mathrm{cfu} / \mathrm{mL}$. Persistence (nonsuccess) was defined as $>20 \mathrm{cfu} / \mathrm{mL}$ of Candida species. Relapse was defined as $20 \mathrm{cfu} / \mathrm{mL}$ of Candida species on day 14 and $20 \mathrm{cfu} / \mathrm{mL}$ on day 42 . Durability of clinical success was evaluated on day 42. Posaconazole was administered to 178 patients and 172 received fluconazole. Clinical success occurred in $91.7 \%$ of posaconazole recipients and in $92.5 \%$ of fluconazole recipients, indicating that posaconazole was not inferior to fluconazole. On day 14, mycologic success was $68 \%$ in both arms, but by day 42 , significantly more posaconazole recipients than fluconazole continued to have mycological success (40.6\% versus $26.4 \%$, respectively). Fewer posaconazole recipients than fluconazole experienced clinical relapse $(31.5 \%$ versus $38.2 \%)$. Adverse effects were similar between the treatment arms. ${ }^{22}$ 
The efficacy and safety of oral posaconazole was evaluated in a Phase III, open-label study by Skiest et al in 2007. ${ }^{11}$ The study consisted of 176 HIV-infected patients with confirmed esophageal candidiasis or OPC who failed previous fluconazole or itraconazole therapy or had laboratory-confirmed resistant mucosal candidiasis. Posaconazole was administered to 103 subjects at $400 \mathrm{mg}$ twice daily for three days, followed by posaconazole $400 \mathrm{mg}$ once daily for 25 days, and 96 patients received posaconazole 400 $\mathrm{mg}$ twice daily for 28 days. There was a clinical response (cure or improvement) in $75.3 \%$ of patients after receiving 28 days of posaconazole treatment. Clinical response rates were similar between both arms of the study. Clinical responses were also similar in different patients with baseline isolates resistant to fluconazole, itraconazole, or both. The most common treatment-related adverse events were diarrhea (11\%), neutropenia (7\%), flatulence $(6 \%)$, and nausea (6\%). Table 1 summarizes the clinical studies with posaconazole in HIV patients.

Few studies have looked at posaconazole as prophylaxis in neutropenic patients. Posaconazole is approved for prophylaxis of emergent filamentous fungi in patients with neutropenia from acute myelogenous leukemia or myelodysplastic syndrome. In a multicenter study published in 2007 by Cornely et al, posaconazole was compared with fluconazole or itraconazole as prophylaxis for invasive fungal infections in patients with prolonged neutropenia. ${ }^{23}$ Three hundred and four patients were randomly assigned to receive posaconazole $200 \mathrm{mg}$ three times daily and 298 patients received fluconazole $400 \mathrm{mg}$ daily or itraconazole $200 \mathrm{mg}$ twice daily. Proven or probable invasive fungal infections were reported in seven patients $(2 \%)$ in the posaconazole group and 25 patients ( $8 \%$ ) in the fluconazole or itraconazole group. The majority of fungal infections were caused by Aspergillus species and there was a clear superiority of posaconazole over fluconazole. Invasive candidiasis occurred in three patients who received posaconazole, in two patients in the fluconazole arm, in none in the itraconazole arm, and the results were not statistically significant. This study showed posaconazole to be more effective in preventing invasive fungal diseases and improving survival.

Another international study by Ullmann et al in 2007 looked at prophylaxis against invasive fungal infections in patients with graft-versus-host disease (GVHD) on immunosuppressive treatment and compared posaconazole with fluconazole. ${ }^{24}$ This was a randomized, double-blind trial including patients who received an allogeneic hematopoietic stem cell transplant and developed GVHD. Posaconazole $200 \mathrm{mg}$ three times daily was administered to 301 patients, and 299 patients received fluconazole $400 \mathrm{mg}$ once daily. Posaconazole was found to be as effective as fluconazole in preventing all invasive fungal infections, but was superior to fluconazole in preventing proven or probable invasive aspergillosis and reducing the rate of deaths related to fungal infections. Colonization with Candida species was assessed and the development of resistance was found to be higher among patients taking fluconazole (17\%) than posaconazole $(5 \%)$.

There have been numerous studies in vitro and in mice showing the potency of posaconazole against different fungi. Pfaller et al compared the new triazole derivatives, ie, posaconazole, ravuconazole, and voriconazole with itraconazole and amphotericin B against 239 clinical isolates of filamentous fungi. All the new azoles had excellent activity against Aspergillus species, posaconazole was better than voriconazole for Rhizopus, and none of them had activity against Fusarium or Mucor, ${ }^{25}$ although other studies showed in vivo activity against a few Fusarium species. ${ }^{26}$

Another study by Sabatelli et al compared the activity of posaconazole with itraconazole, fluconazole, voriconazole, and amphotericin B against 19,000 strains of yeasts and molds. ${ }^{9}$ Posaconazole was more active than, or within 1 dilution of, the comparator drugs against 7000 isolates of Candida and Cryptococcus species. Also, posaconazole was active against species of Candida and Aspergillus which showed resistance to fluconazole, voriconazole,

Table I Evidence for use of posaconazole in HIV patients with OPC and/or esophageal candidiasis

\begin{tabular}{|c|c|c|c|c|c|}
\hline Study & Study design & Study population & Posaconazole & Fluconazole & Results \\
\hline Skiest et al" & $\begin{array}{l}\text { Phase III, } \\
\text { open-label }\end{array}$ & $\begin{array}{l}\text { OPC or EC with } \\
\text { refractory disease } \\
\text { to fluconazole, } \\
199 \text { patients }\end{array}$ & $\begin{array}{l}400 \mathrm{mg} \text { bid for } 3 \text { days, } \\
\text { then } 400 \mathrm{mg} \text { qd for } 25 \text { days } \\
\text { or } 400 \mathrm{mg} \text { bid } \\
\text { for } 28 \text { days }\end{array}$ & & $\begin{array}{l}75 \% \text { clinical } \\
\text { response }\end{array}$ \\
\hline Vasquez et $\mathrm{al}^{22}$ & $\begin{array}{l}\text { Randomized, } \\
\text { evaluator-blinded }\end{array}$ & OPC, 350 patients & $\begin{array}{l}200 \mathrm{mg} \text { first day, then } \\
100 \mathrm{mg} \text { qd for } 13 \text { days }\end{array}$ & $\begin{array}{l}200 \mathrm{mg} \text { first day, then } \\
100 \mathrm{mg} \text { qd for } 13 \text { days }\end{array}$ & $\begin{array}{l}\text { Posaconazole as } \\
\text { effective as } \\
\text { fluconazole, } \mathrm{Cl} 95 \%\end{array}$ \\
\hline
\end{tabular}

Abbreviations: EC, esophageal candidiasis; OPC, oropharyngeal candidiasis; qd, once daily, $\mathrm{Cl}$, confidence interval. 
and amphotericin B, and was much more active than other triazoles against Zygomycetes.

Posaconazole efficacy was studied in mice against C. glabrata isolates with various susceptibilities to fluconazole by Spreghini et al in 2008. ${ }^{27}$ Posaconazole was found to be significantly effective against all the strains.

\section{Posaconazole safety and tolerability}

In a study by Vazquez et al, $64 \%$ of posaconazole therapy recipients (114/178) and 68\% of fluconazole therapy recipients (117/172) reported adverse events during 14 days of OPC treatment. ${ }^{22}$ In the posaconazole and fluconazole treatment groups, only $25 \%$ and $24 \%$ of these events were determined to be posaconazole-related, respectively. The most commonly reported adverse events were diarrhea ( $15 \%$ posaconazole and $20 \%$ fluconazole groups) and nausea (17\% posaconazole and $15 \%$ fluconazole groups). Other reported adverse events $(>5 \%)$ in the posaconazole treatment group included headache (13\%), vomiting (11\%), fever $(10 \%)$, abdominal pain (8\%), and dizziness $(6 \%)$, that were similar to rates reported in the fluconazole treatment arm. Other serious reported adverse events in the posaconazole arm included asthenia, respiratory insufficiency, and lymphadenopathy, but none of these events were associated with posaconazole therapy. ${ }^{22}$

In a study by Skiest et al, adverse events were reported in $49 \%$ of the 199 subjects in the intent-to-treat posaconazole OPC 28-day treatment arms. ${ }^{11}$ Commonly reported adverse events included nausea (12\%), diarrhea (21\%), flatulence $(11 \%)$, abdominal pain $(10 \%)$, headache $(9 \%)$, vomiting $(9 \%)$, dizziness $(7 \%)$, fatigue $(6 \%)$, and rash $(6 \%)$. Neutropenia $(n=28)$ was the most common reported adverse event during posaconazole therapy but only $7 \%$ were possibly or probably associated with posaconazole therapy. $\mathrm{QT}_{\mathrm{c}}$ prolongation, epistaxis, rash, edema, hypersensitivity, vomiting, or laboratory abnormalities led to discontinuation of posaconazole therapy in 11 subjects. ${ }^{11}$ Overall, many of these adverse events are common in HIV-infected or immunocompromised patients due to the disease state and other therapies utilized, including antiretroviral agents and chemotherapy.

While generally well tolerated, patients receiving posaconazole therapy for OPC should be monitored for liver and cardiac dysfunction. Posaconazole should be used with caution in patients with a history or development of adrenal insufficiency, liver dysfunction or failure, and QT interval prolongation. ${ }^{17}$ During posaconazole therapy for OPC, liver function tests including bilirubin levels may rise due to cholestasis. Liver enzymes should be periodically monitored. Serum electrolyte levels, specifically potassium, magnesium, and calcium, should be monitored due to risk of hypocalcemia (9\%), hypokalemia (3\%), and hypomagnesemia (18\%) during posaconazole therapy. As with any medication, patients should be screened prior to use for previous intolerance or contraindications to posaconazole or similar triazole-class agents. $^{17}$

Administration of posaconazole with a cytochrome P450 3A4 substrate should be evaluated prior to start of therapy. Posaconazole has no significant effect on CYP enzymes except CYP3A4. In a study done by Wexler et al, ${ }^{8}$ posaconazole inhibits CYP3A4 at submicromolar concentrations, and other enzymes were not inhibited ( CYP1A2, 2A6, 2C9, 2C19, and 2D6). All azoles inhibit CYP3A4, but the other azoles inhibit some of the other enzymes too, eg, fluconazole and itraconazole inhibit $1 \mathrm{~A} 2$ and $2 \mathrm{C}$, whereas voriconazole inhibits $2 \mathrm{C} 9$ and $2 \mathrm{C} 19$. This means fewer drug inhibitions by posaconazole overall. Contraindications to taking posaconazole are the concurrent use of sirolimus, ergot alkaloids, and the CYP3A4 substrates terbenafine, astemizole, cisapride, pimozine, halofantrine, and quinidine. Caution should be exercised in patients with hypersensitivity to other azoles and potentially proarrhythmic conditions. Serious side effects reported include adrenal insufficiency, cholestasis, hyperbilirubinemia, increased liver enzymes, liver damage, liver failure, and prolonged QT interval. ${ }^{17}$ One study found that adverse effects were not dose-dependent. ${ }^{19}$ Depending on the degree of interaction, alternative therapy or dosage adjustments may be necessary during posaconazole therapy. Additionally, the coadministration of posaconazole and agents with known increased risk of QT prolongation or liver dysfunction should be used with caution. Due to risk of ergotism, administration of ergot alkaloids with posaconazole is contraindicated. Coadministration of posaconazole with acid-blocking agents should be evaluated due to their potential to decrease absorption of posaconazole and therefore decrease serum posaconazole levels during OPC treatment.

\section{Patient satisfaction, acceptability, adherence, and uptake}

Outpatient and inpatient compliance along with patient satisfaction and quality of life with posaconazole OPC therapy is dependent on a variety of factors, including incidence of adverse events, efficacy, dosage frequency, and drug cost. Many of these factors are similar for posaconazole and fluconazole OPC treatment regimens. 
As reported by Skiest et al and Vazquez et al rates of common adverse events such as nausea, vomiting, and diarrhea are similar between fluconazole and posaconazole for OPC therapy. ${ }^{11,22}$ In the study by Vazquez et al, $88 \%$ and $89 \%$ of enrolled patients with OPC completed the treatment phase of posaconazole and fluconazole therapy, respectively, as prescribed. Reasons determined for early discontinuation of therapies included adverse events $(4 \%$ for both treatment groups), treatment failures ( $2 \%$ for both treatment groups), and noncompliance or loss to follow-up, including death (6\% posaconazole group and 5\% fluconazole group). ${ }^{22}$ In the study by Skiest et al, therapy was completed in $75 \%$ of the 199 HIV-infected subjects in an intent-to-treat posaconazole OPC 28-day treatment regimen. Reasons for discontinuation of treatment were adverse events $(23 \%)$, treatment failure $(5 \%)$, non-compliance $(12 \%)$, death $(4 \%)$, and other $(3 \%){ }^{11}$

Based on currently published reports, duration of therapy and dosing schedule is similar for both posaconazole and fluconazole for OPC treatment. Posaconazole OPC treatment regimens generally involve either daily or twice daily dosing. This dosing frequency is significantly less compared with standard posaconazole therapy recommended at three to four times daily for more serious systemic infections. Patient education should include an evaluation of a patient's dietary intake, including timing of dose with meals. Since it is recommended that posaconazole therapy be administered with food, posaconazole frequency may be adjusted in certain patients to maximize compliance of therapy with food intake. While posaconazole therapy has been shown to be efficacious in treatment of OPC, posaconazole therapy may not be feasible, and alternative therapy may need to be evaluated if a patient has difficulty with oral medication absorption due to factors such as significant nausea and vomiting, limited oral intake or use of acid-blocking agents, or gastrointestinal tract dysfunction, including ileus and mucositis.

Whether therapy is prescribed for outpatient or inpatient use, the patient's financial and insurance status may be a barrier to posaconazole therapy. A generic formulation of posaconazole is not currently available in the US, unlike other triazole agents such as fluconazole and itraconazole. Certain insurance companies or institutions may require prior authorization based on formulary restrictions. Additionally, the immediate availability of posaconazole suspension at certain medical centers, clinics, and community pharmacies may be limited and dependent on regional patient populations. Overall, to increase patient compliance with OPC posaconazole therapy, patients should receive information related to the purpose of treatment, directions for administration, and potential side effects of posaconazole therapy.

\section{Conclusions}

Posaconazole is effective in prophylaxis against invasive fungal infections in selected patient populations, as well as for the treatment of some fungi, especially Aspergillus and some Zygomycetes. Posaconazole has been shown to be more effective than fluconazole or itraconazole for prevention of invasive fungal infections in patients with neutropenia from chemotherapy, leukemia, or myelodysplastic syndrome. In the treatment of OPC, posaconazole is at least as effective as fluconazole in HIV patients with OPC. Posaconazole is effective in fluconazole/ itraconazole-resistant OPC and in the treatment of non-OPC fluconazole-resistant Candida species infections. Side effects of posaconazole are similar to fluconazole in most studies, but administration and absorption remain a major concern. Further clinical studies of posaconazole are warranted to extend the range of approved treatment of posaconazole. However, for the vast majority of OPC cases fluconazole remains the drug of choice due to its efficacy and lower cost.

\section{Disclosure}

The authors report no conflicts of interest.

\section{References}

1. Klotz SA, Nand K, Gaur NK, Lipke PN. Oropharyngeal candidiasis: Diagnostic clues, treatment tips. J Respir Dis. 2008;29:128-135.

2. Ruhnke M. Skin and mucous membrane infections. In: Calderone RA, editor. Candida and Candidiasis. Washington, DC: ASM Press; 2002.

3. Sangeorzan JA, Bradley SF, He X, et al. Epidemiology of oral candidiasis in HIV-infected patients: Colonization, infection, treatment, and emergence of fluconazole resistance. Am J Med. 1994;97:339-346.

4. Martinez M, Lopez-Ribot JL, Kirkpatrick WR, Coco BJ, Bachmann SP, Patterson TF. Replacement of Candida albicans with C. dubliniensis in human immunodeficiency virus-infected patients with oropharyngeal candidiasis treated with fluconazole. J Clin Microbiol. 2002;40: 3135-3139.

5. Coker RJ, Fisher M, Tomlinson DR. Management of mycosis associated with HIV disease. Int J STD AIDS. 1995;6:408-412.

6. Pappas PG, Kauffman CA, Andes D, et al. Clinical practice guidelines for the management of candidiasis: 2009 update by the Infectious Diseases Society of America. Clin Infect Dis. 2009;48:503-535.

7. Munayyer HK, Mann PA, Chau AS, et al. Posaconazole is a potent inhibitor of sterol 14-alpha demethylation in yeasts and molds. Antimicrob Agents Chemother. 2004;48:3690-3696.

8. Wexler D, Courtney R, Richards W, et al. Effect of posaconazole on cytochrome P450 enzymes: A randomized, open-label, two-way crossover study. Eur J Pharm Sci. 2004;21:645-653.

9. Sabatelli F, Patel R, Mann PA, et al. In vitro activities of posaconazole, fluconazole, itraconazole, voriconazole, amphotericin B against a large collection of clinical important molds and yeasts. Antimicrob Agents Chemother. 2006;50:2009-2015.

10. Schiller DS, Fung HB. Posaconazole: An extended-spectrum triazole antifungal agent. Clin Ther. 2009;29:1862-1886.

11. Skiest DJ, Vazquez JA, Anstead GM, et al. Posaconazole for the treatment of azole refractory oropharyngeal and esophageal candidiasis in subjects with HIV infections. Clin Infect Dis. 2007;44:607-614. 
12. Rex JH, Rinaldi MG, Pfaller MA. Resistance of Candida species to fluconazole. Antimicrob Agents Chemother. 1995;39:1-7.

13. National Committee for Clinical Laboratory Standards. Reference method for broth dilution antifungal susceptibility testing of yeasts: Proposed standard. Villanova, PA: NCCLS; 2008.

14. Revankar SG, Kirkpatrick WR, McAtee RK, et al. Detection and significance of fluconazole resistance in oropharyngeal candidiasis in human immunodeficiency virus-infected patients. J Infect Dis. 1996; 174:821-827.

15. Xiao L, MadisonV, Chau AS, et al. Three-dimensional models of wild-type and mutated forms of cythochrome P450 14-alpha sterol demethylases from Aspergillus fumigatus and Candida albicans provides insights into posaconazole binding. Antimicrob Agents Chemother. 2004; 48:568-574.

16. Pfaller MA, Messer SA, Boyken L, et al. In vitro activities of voriconazole, posaconazole, and fluconazole against 4169 clinical isolates of Candida spp. and Cryptococcus neoformans collected during 2001 and 2002 in the ARTEMIS global antifungal surveillance program. Diagn Microb Infect Dis. 2004;48:201-205.

17. Noxafil prescribing information. Kenilworth, NJ: Schering Corporation; 2008.

18. Courtney R, Sansone A, Smith W, et al. Posaconazole pharmacokinetics, safety and tolerability in subjects with varying degree of chronic renal disease. J Clin Pharmacol. 2005;45:185-192.

19. Courtney R, Pai S, Laughlin M, Lim J, Batra V. Pharmacokinetics, safety, and tolerability of oral posaconazole administered in a single and multiple doses in healthy adults. Antimicrob Agents Chemother. 2003;47:2788-2795
20. Krishna G, Moton A, Ma L, et al. The pharmacokinetics and absorption of posaconazole oral suspension under various gastric conditions in healthy volunteers. Antimicrob Agents Chemother. 2009;53:958-966.

21. Herbrecht R. Posaconazole: A potent, extended-spectrum triazole anti-fungal for the treatment of serious fungal infections. Int $J$ Clin Pract. 2004;58:612-624.

22. Vasquez JA, Skiest DJ, Nieto L, et al. A multicenter randomized trial evaluating posaconazole versus fluconazole for the treatment of oropharyngeal candidiasis in subjects with HIV/AIDS. Clin Infect Dis. 2006;42:1179-1186.

23. Cornelly OA, Maertens J, Winston D, et al. Posaconazole vs fluconazole or itraconazole prophylaxis in patients with neutropenia. $N$ Engl J Med. 2007;356:348-359.

24. Ullmann AJ, Lipton JH, Vesole DH, et al. Posaconazole or fluconazole for prophylaxis in severe graft-versus-host disease. NEngl J Med. 2007; 356:335-347.

25. Pfaller MA, Messer SA, Hollis RJ, Jones RN; Sentry Participants Group Antifungal activities of posaconazole, ravuconazole, and voriconazole compared to those of itraconazole and amphotericin B against 239 clinical isolates of Aspergillus spp. and other filamentous fungi: Report from SENTRY antimicrobial surveillance program. Antimicrob Agents Chemother. 2002;46:1032-1037.

26. Sponsel WE, Graybill JR, Nevarez HL, et al. Ocular and systemic posaconazole treatment of invasive Fusarium solani keratitis and endophthalmitis. Br J Ophthalmol. 2002;86:829-830.

27. Spreghini E, Maida CM, Tomasetti S, et al. Posaconazole against Candida glabrata isolates with various susceptibility to fluconazole. Antimicrob Agents Chemother. 2008;52:1929-1933.
Infection and Drug Resistance

\section{Publish your work in this journal}

Infection and Drug Resistance is an international, peer-reviewed openaccess journal that focuses on the optimal treatment of infection (bacterial, fungal and viral) and the development and institution of preventive strategies to minimize the development and spread of resistance. The journal is specifically concerned with the epidemiology of antibiotic

\section{Dovepress}

resistance and the mechanisms of resistance development and diffusion in both hospitals and the community. The manuscript management system is completely online and includes a very quick and fair peerreview system, which is all easy to use. Visit http://www.dovepress.com/ testimonials.php to read real quotes from published authors. 